Service social

\title{
Les défis d'une interface : les groupes de femmes et le réseau de la santé et des services sociaux de la région de Québec
}

\section{Marie-Andrée Couillard et Ginette Côté}

Volume 42, numéro 2, 1993

Recherche et pensée critique

URI : https://id.erudit.org/iderudit/706616ar

DOI : https://doi.org/10.7202/706616ar

Aller au sommaire du numéro

Éditeur(s)

École de service social de l'Université Laval

ISSN

1708-1734 (numérique)

Découvrir la revue

Citer cet article

Couillard, M.-A. \& Côté, G. (1993). Les défis d'une interface : les groupes de femmes et le réseau de la santé et des services sociaux de la région de Québec. Service social, 42(2), 29-49. https://doi.org/10.7202/706616ar
Résumé de l'article

Les propositions de la réforme Côté en ce qui concerne le communautaire ne sont pas sans susciter des inquiétudes du point de vue des groupes de femmes. Nous voulons montrer que ces groupes ne constituent pas un ensemble homogène, qu'ils représentent des intérêts différents qui se manifestent clairement lorsqu'ils s'engagent dans des processus de concertation. Ils entretiennent de plus des liens inter-organisationnels complexes, notamment par la participation à des tables de concertation. Cette participation soulève des paradoxes et entraîne des effets non prévus, dont les auteures font état. Enfin, le roulement dans le personnel bénévole et la clientèle affecte parfois leur enracinement. En somme, il apparaît que la concertation exigée par le partenariat doit être sans cesse renégociée et ne peut en aucun cas constituer le point de départ d'un processus d'arrimage aux institutions étatiques. 


\section{Les défis d'une interface:}

\section{les groupes de femmes et le réseau \\ de la santé et des services sociaux \\ de la région de Québec}

Marie-Andrée COUILLARD
Professeure
Département d'anthropologie
Université Laval
Ginette CÔTÉ
Professionnelle de recherche

Centre de recherche sur les services communautaires

Université Laval

\section{INTRODUCTION}

L'existence d'une « interface » entre le communautaire et le réseau de la santé et des services sociaux a déjà fait l'objet d'études, surtout depuis le dépôt du rapport Rochon (1988). L'expression « interface » a d'ailleurs déjà été utilisée par Lamoureux et Lesemann (1987) pour nommer ce point de contact.

1. L'analyse présentée ici a déjà fait l'objet d'une communication dans le cadre du colloque du Centre de recherche sur les services communautaires: Au carrefour de la recherche et des pratiques dans les services sociaux et communautaires, en octobre 1992. Nous désirons remercier mesdames Jacqueline Fournier, Colette Lavoie et Sandra Shee ainsi que messieurs Yves Hurtubise et Hector Ouellet pour leurs commentaires pertinents. 
Nous reprenons ici cette notion qui, en informatique, désigne l'espace commun à deux ensembles, pour faire référence à un espace social dans lequel des acteurs aux intérêts variés se déploient.

Les relations entre l'État et le mouvement communautaire se sont transformées profondément depuis les années 60. Les groupes populaires ont bâti leurs discours et entamé leurs actions dans un contexte de contestation face à l'État, alors que maintenant s'amorce une période de compromis pendant laquelle leurs propos et leurs pratiques semblent se rapprocher de plus en plus de celui qui jadis jouait le rôle de l'ennemi. Selon Larochelle et Gilbert (1991), les différentes analyses concernant ces transformations s'organisent autour de deux pôles. Le premier s'appuie sur une vision passéiste qui incite certains partisans à déplorer l'absence d'une contestation plus politique, considérée comme le germe du changement social, au profit de valeurs et d'intérêts d'ordre plus économique qui leur paraissent moins nobles. Le second pôle regroupe les analyses qui optent pour une vision plus constructive du présent et qui postulent une recomposition du social en s'inspirant du potentiel créateur des groupes, perçu comme une source de renouveau.

Notre contribution s'inscrit dans cette perspective pragmatique dans la mesure où elle vise surtout à faire un constat en partant du point de vue des groupes de femmes en interaction avec le réseau de la santé et des services sociaux de la région de Québec. Une revue des écrits nous permet de définir le problème en situant certains effets appréhendés de la réforme Côté, en clarifiant la notion de partenariat entre l'État et les groupes communautaires et, enfin, en définissant ce que nous entendons par groupes de femmes. Suit une synthèse des principaux défis que pose la réussite de partenariats entre les groupes de femmes de la région de Québec et les CLSC. Cette synthèse s'appuie sur un ensemble complexe de données recueillies au cours d'une enquête qualitative menée depuis $1990^{2}$.

\section{LA CONJONCTURE ACTUELLE DANS LES ÉCRITS}

La problématique qui organise cette synthèse résulte de la recension d'une série d'écrits portant sur différents aspects du rapport entre le communautaire

2. Cette recherche, financée par le Conseil de la recherche en sciences humaines du Canada (CRSHC) pour une durée de trois ans, a débuté en août 1990 (Couillard et Côté, 1992, 1991 ; Côté, 1991 ; Couillard, à paraître ; Couillard, 1990). L'étude a pour thème central "Les stratégies de pouvoir dans les organisations féminines ». L'objectif principal n'est pas de voir comment les femmes ont du pouvoir « sur », mais plutôt de documenter de l'intérieur et d'analyser 1) comment elles voient le pouvoir, 2) comment se fait le partage du pouvoir à l'interne, 3) comment elles se donnent du pouvoir face aux autres groupes et éventuellement face à l'État. Sans être au cœur des préoccupations de cette recherche, les liens entre les groupes de femmes et l'État ont eux aussi fait l'objet d'une collecte de données. 
et l'État, émanant surtout de chercheurs formés en service social ou en sociologie et, dans une moindre mesure, en psychologie, mais aussi de personnes directement engagées dans l'action communautaire ${ }^{3}$.

\section{Les groupes communautaires et la réforme Côté}

Un constat s'impose avec force dans les écrits consultés: avec la réforme Côté, tout porte à croire que le réseau englobera désormais les organismes communautaires, pensés désormais comme son extension «naturelle». C'est pourquoi l'un des enjeux majeurs de cette réforme sera la consolidation des rapports entre les CLSC et les groupes communautaires, comme le mentionne M. Sénéchal, de la Fédération des CLSC, qui souligne la nécessité d'une meilleure compréhension de ce phénomène (dans Fournier, 1991 : 11).

Cette nouvelle loi sur la santé et les services sociaux s'inscrit dans la foulée d'un processus de réflexion amorcé vers la fin des années 80. Plusieurs rapports importants ont alimenté cette réflexion ${ }^{4}$, mais c'est le rapport Rochon (1988) que l'on considère comme la source d'inspiration de la réforme tout entière.

La Commission d'enquête sur les services de santé et les services sociaux ${ }^{5}$ (la Commission Rochon) a été créée en 1985. Déjà, la participation des organismes communautaires à cette commission a été très importante, puisque ces organismes ont à eux seuls présenté 37,5 \% des mémoires (Robert, 1989: 45) dont une bonne partie provenait des groupes de femmes. Leur grande participation a aussi été constatée à la Commission parlementaire organisée par le ministre Côté à l'hiver 1990. Et depuis les dépôts successifs du Livre blanc, du projet de loi 120 et des documents connexes, ces organismes ont, sans relâche, fourni commentaires et avis sur les aspects les concernant et notamment sur les liens les unissant ou devant les unir à l'État.

3. Notre propos s'inscrit dans la tradition anthropologique qui privilégie une approche qualitative tout en s'appuyant sur une perspective comparative, sinon dans le traitement des données, du moins dans la conceptualisation du problème. Notre objectif est de traduire les référents socioculturels des acteurs sociaux afin de les rendre plus accessibles à d'autres et éventuellement de révéler la logique qui organise leurs actions. Nous nous situons donc en marge des préoccupations des intervenants, tant du réseau officiel que du communautaire.

4. Le rapport Harnois (1987) sur la santé mentale, le rapport Brunet (1987) sur les activités et services des CLSC et le rapport Harvey (1991) sur la Direction de la protection de la jeunesse ont tous trois donné lieu à des orientations ou à des réorganisations importantes dans leurs domaines respectifs.

5. Le mandat de la Commission consistait à étudier les objectifs, le fonctionnement, le financement et le développement du système de services sociaux (Commission d'enquête sur la santé et les services sociaux, 1988 : i). 
Un examen de la loi, de la politique de santé et des modalités de financement proposées nous fait découvrir que la reconnaissance des organismes communautaires constitue l'une des innovations majeures de la réforme. Cette reconnaissance s'inscrit dans la loi $^{6}$, certains articles y étant consacrés (en particulier les articles 334 à 336, Assemblée nationale, 1991 : 97). Elle se manifeste aussi par la promesse d'un financement plus adéquat qui ajoute de la substance au nouveau statut des organismes communautaires, élevés au rang de partenaires. Enfin, cette reconnaissance passe par une volonté accrue de l'État de favoriser une plus grande participation de ce type d'organismes tant dans la prestation de services que dans la prise de décision.

En ce sens, notons que l'article 421 leur accorde $20 \%$ des sièges aux assemblées régionales ${ }^{7}$. Ces dernières ont élu en avril 1993 les représentants aux conseils d'administration des régies régionales en tenant compte de ce pourcentage. Ces régies ont pour mandat, entre autres, de planifier, d'évaluer et d'assurer la coordination des services pour chacune des régions en tenant compte du critère de complémentarité. En théorie, tout doit donc passer par la régie qui élabore les plans régionaux d'organisation de services (PROS) en collaboration étroite avec des représentants en provenance du milieu communautaire.

Fait à noter, les groupes de femmes ne font pas l'objet de traitement spécial dans cette réforme ${ }^{8}$. Déjà en 1990, au moment de la consultation de la Commission des affaires sociales, des groupes signalent que les problèmes particuliers aux femmes sont singulièrement absents de la réforme (David, 1991 : 6). Un an plus tard, le Conseil du statut de la femme arrive à la même conclusion et propose une série de recommandations afin que la spécificité des femmes, à la fois comme clientèle et comme professionnelles et bénévoles dans le champ de la santé et des services sociaux, soit prise en considération. Ces recommandations n'ont guère eu d'écho dans l'ensemble de la réforme et l'on déplore toujours le fait qu'aucune mesure ne soit prise pour qu'elles aient voix au chapitre, en dépit du fait qu'elles ont recours deux fois plus souvent aux services de santé et aux services sociaux (David, 1991; Latérière et Voyer, 1991). À cet égard, soulignons l'absence du principe

6. "Dans la présente loi, on entend par "organisme communautaire" une personne morale constituée en vertu d'une loi du Québec à des fins non lucratives dont les affaires sont administrées par un conseil d'administration composé majoritairement d'utilisateurs des services de l'organisme ou de membres de la communauté qu'il dessert et dont les activités sont reliées au domaine de la santé et des services sociaux » (Assemblée nationale, 1991 : 97).

7. Les assemblées régionales sont composées de 150 membres dont $40 \%$ proviennent des établissements, $20 \%$ des groupes communautaires, $20 \%$ des groupes socio-économiques et $20 \%$ des municipalités (Assemblée nationale, $1991: 118-119$ ).

8. Dans les régies régionales, par exemple, on ne s'est pas assuré que les groupes de femmes auraient des représentantes. 
d'égalité dans la représentation des sexes aux instances décisionnelles que sont les conseils d'administration des établissements et les régies régionales; cette revendication a pourtant été maintes fois formulée tant par le CSF que par les groupes de femmes.

Devant les nouvelles règles du jeu imposées par l'État, les réactions du communautaire et, encore une fois, celles en provenance des groupes des femmes, furent immédiates et secondées par les commentaires de chercheurs engagés à différents niveaux dans l'action communautaire. Certains s'allient à la position de Bozzini (1990) à la suite du rapport Rochon et insistent sur les brèches intéressantes à exploiter pour le communautaire. Deslauriers (1991) et Robert (1989) mentionnent tous deux que les régies régionales, par exemple, permettront une plus grande visibilité pour les organismes et qu'il se créera là un espace pour faire des alliances. Selon White, tout n'est pas si simple. La réforme entraînera soit une nouvelle effervescence, soit « ...une disparition du communautaire étouffé par trop d'encadrement» (citée dans Fournier, 1991: 11).

En dépit de la reconnaissance de certains avantages de la réforme pour les organismes communautaires, la majorité des commentateurs dénoncent ses effets négatifs potentiels ${ }^{9}$. Ce qui effraie particulièrement, c'est l'idée « d'intégration au système » officiel. On souligne la peur d'être harnaché au réseau, d'en être réduit à accomplir uniquement un travail de sous-traitance. Trottier (1991) craint pour l'intégrité de l'intervention communautaire advenant une complémentarité forcée. Parazelli (1990), quant à lui, nous met en garde contre une standardisation des pratiques résultant d'un encadrement étatique trop serré. D'autres manifestent de la méfiance devant l'ingérence additionnelle d'une logique bureaucratique et technocratique, au sens de Lamoureux et Lesemann (1987). De plus, plusieurs ont noté que l'approche en fonction de groupes cibles et de programmes cadres n'est pas compatible avec l'approche globale et les objectifs d'action sociale des organismes communautaires. Enfin, plusieurs soulignent qu'il s'agit en fait d'une façon pour le réseau de procurer des services à la population à moindres coûts, dans un contexte de désistement d'un État néo-libéral qui s'accommode parfaitement de la logique médicale à l'égard des problèmes de santé et des problèmes sociaux (Caillouette, 1992; David, 1991; Guay, 1991; Table des organismes communautaires et bénévoles, 1991).

Deux autres éléments importants de la réforme qui auront un impact sur les groupes communautaires ont échappé aux critiques. Premièrement, il est dorénavant permis de déposer des plaintes concernant les services offerts par les groupes communautaires (Assemblée nationale, 1991 : 22). Deuxième-

9. Au moment où ce texte a été révisé pour publication, l'implantation de la réforme, depuis avril 1993, n'avait pas encore fait l'objet d'évaluation. 
ment, la loi reconnaît un organisme communautaire seulement dans la mesure où ses affaires sont administrées par un conseil d'administration (article 334). Or, plusieurs groupes communautaires et en particulier les groupes de femmes s'organisent plutôt sur le mode du collectif assumant la gestion des affaires du groupe sur une base égalitaire. Un tel mode d'organisation ne sera plus acceptable selon les termes de la loi.

Les représentants de la Table de concertation des organismes communautaires et bénévoles (1991: 3) ont donc raison de souligner que cette loi s'appuie sur une contradiction fondamentale: d'une part, elle affirme l'autonomie du communautaire et, $d^{\prime}$ autre part, elle impose du même souffle des modalités très strictes d'intégration au système de services publics. Cette tension entre l'autonomie et l'intégration se trouvera de plus en plus mise à l'épreuve dans le cadre du partenariat tel que redéfini par la loi.

\section{Le partenariat}

Comme le souligne Guay (1991), la régionalisation, de nouveau en vogue au Québec depuis une dizaine d'années, nous force dans l'ère du partenariat. Robert (1989: 45) nous dit à cet effet que «De l'anti-étatisme de gauche durant les années 70 et de droite des années 70-80, on est en train de passer à une conception de l'État partenaire de la société civile ». La création des conseils régionaux de développement, qui allient des représentants de I'entreprise privée, de la communauté et de l'État, est une illustration de ce nouveau type de partenariat dans le domaine économique (Fournier, 1991; Fournier et Bourque, 1990; Robert, 1989). Dans le domaine de la santé et des services sociaux, le partenariat entre organismes communautaires et bénévoles et les institutions s'était déjà amorcé dans les CRSSS, notamment avec les PROS en santé mentale et les programmes de maintien à domicile. II promet de s'intensifier maintenant qu'ils sont devenus des régies régionales.

Les travaux portant sur l'état des relations entre le communautaire et l'État se sont développés surtout vers la fin des années 80 avec ceux de la commission Rochon ${ }^{10}$. Les auteurs qui ont traité ce thème ont utilisé différents termes pour désigner les liens tissés entre les groupes communautaires et l'État. Ainsi les notions de «collaboration», de «concertation » et d' « interface» sont souvent employées de manière indifférenciée, révélant du même coup

10. Par exemple, les études bien connues de Lamoureux et Lesemann sur Les filières de I'action sociale... (1987), de Godbout et al. éclairant La face cachée du système (1987), de Bélanger sur La concertation dans le réseau des services de santé et des services sociaux (1987), de Vaillancourt et al. concernant La privatisation des services sociaux (1987), ou celles plus récentes de Dumais portant sur Les CLSC et les groupes communautaires en santé... (1991) et de Panet-Raymond et Bourque posant la question du Partenariat ou pater-nariat? (1991). 
une certaine opacité et un flottement conceptuel qui fait s'équivaloir différents niveaux de formalisation des pratiques et des modes de négociation entre des parties.

Pour tenter de contourner ce flou conceptuel, Panet-Raymond et Bourque (1991 : 9-10) présentent les pratiques de collaboration comme s'étalant sur un continuum en fonction de leur nature égalitaire ou inégalitaire. Cependant, il faut souligner que la littérature insiste plutôt sur les aspects inégalitaires des relations entre les organismes communautaires et I'État. L'étude de Dumais (1991 : 30) démontre bien qu'en dépit des affirmations des CLSC, qui se disent engagés avec les groupes communautaires en santé notamment, la collaboration est plus étroite lorsqu'il s'agit de prêt d'équipement ou d'allocation de budget que lorsqu'elle nécessite l'apport des ressources humaines.

Exception faite des appuis matériels (infrastructure), c'est pour la référence de clients que les relations les plus fréquentes entre les organismes communautaires et l'État sont créées (Godbout et al., 1987 : 70). C'est ce que le communautaire appelle le «dumping » de clientèle, processus qui lui donne l'impression d'être un déversoir pour les établissements qui ne peuvent ou ne veulent pas assumer un trop-plein de clientèle (Lamoureux et Lesemann, 1987: 144).

L'élaboration de programmes communs avec l'une ou l'autre des instances du réseau n'a pas donné lieu à des rapports plus symétriques. En guise d'exemple, il faut voir la verte critique de Lorraine Guay (1991: 9-10) à l'égard de certains PROS en santé mentale. Selon elle, les CRSSS se sont avérés les tout-puissants « $4^{\mathrm{es}}$ tiers », ce qui se manifeste en particulier par une absence de débat de fond.

Les auteurs s'accordent donc pour dire que le tableau des relations entre les groupes communautaires et l'État est assez sombre. Des ententes « exceptionnelles » s'inscrivent cependant au chapitre des réussites. C'est probablement ce qui justifie l'énumération que font Panet-Raymond et Bourque (1991 : 12-14) des facteurs liés à la réussite du partenariat. Selon eux, ces facteurs sont de trois ordres: organisationnels, professionnels et humains. De cette lecture, nous retenons que ce sont l'ouverture et le respect envers I'autre qui seraient les facteurs déterminants de la réussite du partenariat.

\section{Les groupes de femmes}

L'ouverture et le respect sont, il va sans dire, des attitudes on ne peut plus désirables dans une perspective de concertation. Il faut cependant se demander dans quels contextes ces deux attitudes doivent effectivement se déployer, notamment en ce qui concerne les groupes de femmes qui retiennent notre attention ici. 
Les groupes de femmes sont aussi désignés dans la littérature par les expressions « organisations », « associations » ou " groupements » de femmes. De Home (1987 : 18 citant Hare) on retient qu'il s'agit d'un rassemblement de personnes qui interagissent face à face, qui partagent des objectifs et des règles régissant leurs activités de groupe et qui choisissent des façons d'établir des rapports mutuels qui les distinguent d'autres groupes. Ouellette (1986: 2) souligne pour sa part que les groupes de femmes sont des acteurs sociaux mobilisés pour influencer les conditions de vie des femmes.

Dans une perspective historique, le collectif Clio (Dumont et al., 1992), Monet-Chartrand (1990) et Home (1987) explorent le développement des organisations féminines et leur lien avec le mouvement féministe, alors que I'ouvrage de Cohen (1990) documente l'œuvre des Cercles de fermières, et celui de Lamoureux, Gélinas et Tari (1993) l'évolution de l'Association féminine d'éducation et d'action sociale (AFEAS).

De son côté, le Conseil du statut de la femme a déjà recensé l'ensemble des groupements féminins du Québec au cours d'une vaste enquête qui sert d'arrière-fond à notre étude (Ouellette, 1986). Cette enquête visait à documenter l'action des groupes, leurs modes d'intervention, leurs activités, leur structuration (au sens formel), leur financement et leurs liens avec d'autres groupes. Parce qu'elle se voulait quantitative, cette étude n'aborde pas, elle non plus, les rapports fins entre les groupes et les institutions du réseau de la santé et des services sociaux tels qu'ils se structurent dans des pratiques usuelles. Il en est de même des travaux de Anadon et al. $(1992,1990)$ et de Masson, Tremblay et Tremblay (1989) qui, bien qu'ils couvrent plusieurs aspects des groupes de femmes en région, abordant à la fois l'idéologie qui les inspire et les pratiques qui les caractérisent, ne se centrent pas sur leur articulation à l'État.

\section{À PROPOS DU PROBLÈME ET DES DONNÉES}

En résumé, nous avons vu que l'espace social entre l'État et le communautaire se rétrécit sous l'impact de la réforme Côté. Les groupes de femmes, partie prenante de l'univers communautaire depuis des décennies, se font entendre avec force. Certains se verront désormais reconnus par la loi comme une extension «naturelle» du réseau. Pour plusieurs, leur financement, et parfois leur existence, dépend de cette reconnaissance. Ce constat pose de façon aiguë la question du partenariat à instaurer, de sa nature et de ses effets sur l'ensemble des groupes. Nous avons postulé que ce partenariat se noue dans un espace désigné par l'expression interface. Le recours à cette notion évite l'ambiguiité du terme partenariat que nous réservons pour désigner des discours ou des pratiques mettant en scène des instances particulières (un CLSC et un Centre de femmes par exemple). Il paraît opportun, maintenant, 
de brosser un portrait des principaux défis que pose l'existence de cette interface, pour la réalisation de partenariats tels que souhaités par l'État, d'une part, et, d'autre part, pour le mouvement des femmes et sa base communautaire.

Ce portrait prend la forme d'une synthèse en trois points, produit d'une première analyse des données recueillies au cours des trois dernières années selon une approche qualitative relevant de l'anthropologie. Notons d'abord que les données n'ont pas été recueillies pour répondre aux interrogations soulevées ici, mais bien en fonction d'une autre problématique dont les ramifications nous ont amenées à considérer la question du rapport à l'État. $C^{\prime}$ est à la lumière de cette incursion volontaire dans une littérature nouvelle que nous avons revu nos données. Il ne s'agit donc pas ici de faire état des grilles qui ont permis de recueillir l'information nécessaire à l'élaboration de l'analyse qui suit.

Précisons cependant que c'est par l'observation participante auprès d'une dizaine de groupes de femmes, l'engagement actif dans les activités d'une Table de concertation de la région de Québec (03), le suivi de rencontres annuelles de certains regroupements provinciaux, la participation ad hoc à des colloques organisés par des groupes et à d'autres tables de concertation que nous avons recueilli une masse considérable de données notamment sur la nature des groupes en présence, sur leurs liens inter-organisationnels ${ }^{11}$ et sur les itinéraires des personnes qui y œuvrent. Quatre auxiliaires étudiantes (pendant un an), une professionnelle de recherche et la responsable du projet ont été directement engagées dans le processus. De plus, trente entrevues semi-directives ont permis de mieux cerner certains aspects des processus qui nous intéressent, en particulier ceux qui exigaient une connaissance plus approfondie des personnes concernées afin de conserver à la réalité étudiée toute sa complexité ${ }^{12}$.

Les groupes choisis pour l'observation participante l'ont été, non pas en fonction de leur appartenance au mouvement féministe au sens strict, mais plutôt parce que leurs pratiques constituent autant de lieux, créés par des femmes, pour agir sur leur condition. Ainsi, nous avons retenu toutes les

11. Dans la mesure du possible, pour chacun des groupes à l'étude, un « dossier inter-organisationnel » a été monté à leur intention. Ce travail faisait partie de notre contribution bénévole, une prestation offerte en contrepartie de la permission de nous intégrer aux activités du groupe. De l'avis de certains groupes, en plus de révéler une partie du travail rarement comptabilisé et valorisé, ce dossier leur est dorénavant fort utile lors des demandes de subventions, notamment dans les sections se rapportant aux liens avec le milieu.

12. Soulignons que, selon le protocole anthropologique, nous sommes liées par notre obligation de préserver l'anonymat de nos informatrices. La région de Québec étant ce qu'elle est, toute allusion à des pratiques propres à certains groupes aurait pour effet de les identifier aux yeux des gens qui les connaissent. Nous devons donc traiter nos informations avec la plus grande prudence, la synthèse étant ici un moyen d'éviter les détails ethnographiques révélateurs. 
stratégies d'action: celles qui s'identifient au féminisme radical constituant un extrême, et les œuvres des religieuses, I'autre. C'est, dans les faits, cet ensemble complexe qui constitue le communautaire lorsqu'on fait référence aux groupes de femmes.

\section{LES DÉFIS DE L'INTERFACE}

Toute démarche qui vise la compréhension de l'interface entre le communautaire et le réseau doit tenir compte de cette complexité. Les groupes de femmes se présentent dans cet espace avec une histoire et des pratiques qui leur sont propres et nous allons suggérer que les pressions résultant de l'exercice du partenariat tel que pensé dans la réforme Côté vont avoir des effets à long terme sur l'ensemble des groupes et, de là, sur le mouvement des femmes. Trois éléments sont retenus comme porte d'entrée dans ce débat: la nécessaire hétérogénéité des groupes en présence; la diversité des liens inter-organisationnels qu'ils entretiennent et le roulement constant de leurs effectifs et de leurs clientèles.

\section{L'hétérogénéité}

Le communautaire peut paraître relativement homogène si nous l'abordons de l'extérieur en postulant que les groupes qui le composent partagent une culture, une identité ou un mode d'intervention. Il devient alors plus simple de l'opposer à un État dont les pratiques, les valeurs et les intérêts seraient radicalement différents. Or, voilà que la réalité des groupes de femmes, partie intégrante du communautaire, se révèle tout autre. Bien sûr, vus de l'extérieur, les groupes de femmes ont des points communs: tous agissent afin d'améliorer la condition des femmes; tous ont recours au travail bénévole qui suppose un certain don de soi; tous honnissent le morcellement de l'individu selon ses besoins et ses problèmes. Ces points communs constituent d'ailleurs le fondement d'une solidarité dynamique. Tout intervenant de l'extérieur doit donc faire preuve d'ouverture et de respect face aux choix qu'ont faits ces femmes engagées. Mais ayant dit cela, il nous semble que nous n'avons fait qu'effleurer la réalité.

Car, à un autre niveau, le communautaire c'est aussi un lieu d'expérimentation, d'éclatement, de dissonances et de conflits. II faut alors se demander qui, dans ce contexte, doit être ciblé comme partenaire et se voir attribuer une légitimité fort utile en période de compétition pour des ressources de plus en plus limitées. Il faut aussi prévoir les conséquences qu'auront ces choix sur les autres groupes dès lors marginalisés parce que non reconnus. Pour y parvenir, nous devons d'abord saisir ce qui semble être générateur de différences. 
Les groupes de femmes sont multiples et hétérogènes, nous l'avons dit. Cette hétérogénéité découle de plusieurs facteurs, notamment la vocation, impliquant différents types d'activités et de services offerts, les approches, le mode d'organisation, la formation et la motivation des ressources humaines, les sources de financement et l'origine du groupe.

D'après nous, la vocation impliquant différents types d'activités et de services offerts par les groupes constitue le premier critère de leur différenciation. Certains sont à vocation spécifique, c'est-à-dire qu'ils se centrent sur une problématique (la défense des droits des femmes assistées sociales, par exemple), tandis que d'autres se distinguent par leur polyvalence. Parmi cet ensemble, des groupes de femmes offrent des services directs comme de l'hébergement pour les femmes victimes de violence, alors que d'autres organismes sont plus axés sur la référence indirecte et de l'information téléphonique. La variété des activités et des services offerts signifie qu'ils touchent à leur tour différentes clientèles, elles-mêmes jugées plus ou moins prioritaires par le ministère de la Santé et des Services sociaux.

Ce critère, en apparence sans conséquence, a pourtant son importance pour quiconque souhaite établir un partenariat dans le cadre de la réforme Côté. Ainsi, il y a fort à parier que les relations entre le réseau et les groupes de services à vocation spécifique ou ceux qui offrent des services directs et qui sont largement financés par l'État vont s'intensifier, s'inscrivant ainsi dans la perspective de complémentarité souhaitée par la réforme. Bien avant l'avènement de cette réforme, on constatait déjà un resserrement dans la marge de manœuvre de bien des groupes, phénomène qui ira en grandissant au fur et à mesure de son implantation. Certains groupes vont par conséquent se voir attribuer une légitimité accrue, du moins du point de vue du réseau. Un des défis importants sera alors le respect de la mission que ces groupes se sont eux-mêmes donnée. L'intervention directe de l'État dans leur programmation afin d'ajouter un ou des mandats particuliers (par exemple, si le CLSC demande à un Centre de femmes de s'impliquer dans le maintien à domicile) les amènerait facilement à compromettre leur identité et leur autonomie. De plus, cette ingérence pourrait nuire au maintien d'une interface fructueuse en instaurant un rapport de force par l'imposition directe $d$ 'une logique bureaucratique dans un espace alternatif.

Un deuxième critère de différenciation renvoie au type d'approche préconisé et soutenu par chacun des groupes. Bien qu'il soit tentant de penser que I'approche féministe (ou l'intervention féministe) constitue un critère d'homogénéité entre les groupes, il en va tout autrement dans la réalité. Dans les faits, certains groupes adhèrent à un féminisme qualifié de radical, d'autres optent pour un féminisme plus souple, alors que d'autres encore hésitent à utiliser le terme en affirmant que ce qui compte, c'est de «travailler pour que la femme soit heureuse». 
Cette diversité d'approches pose un autre défi à la collaboration entre les groupes de femmes et le réseau. Les intervenantes des groupes et celles issues du CLSC ne se situent pas obligatoirement dans la même perspective, elles ne partagent pas nécessairement les mêmes valeurs et les mêmes idéaux politiques. Certaines femmes se sentent investies $d^{\prime}$ 'une mission et veulent «sauver» les autres, d'autres croient en leur potentiel et veulent les rendre autonomes, d'autres doivent atteindre des objectifs particuliers découlant de politiques qui leur sont parfois étrangères. Enfin, certaines naviguent entre ces différentes tendances selon les moments et les lieux.

Encore une fois, toute action du réseau dans les groupes peut avoir un effet déséquilibrant en donnant à des approches plutôt qu'à d'autres une légitimité qui, elle, risque d'entraîner la généralisation d'un vocabulaire (féministe par exemple) sans contrepartie réelle sur le plan de l'action (notamment en ce qui concerne la remise en question des rapports de pouvoir). En s'adaptant ainsi à une certaine façon de définir la réalité des femmes, ce discours brouille les enjeux réels et rend la réalisation d'un partenariat d'autant plus difficile.

Le mode d'organisation introduit lui aussi des variations importantes dans la configuration des groupes de femmes. La plupart définissent d'une manière qui leur est propre leur rapport à l'autorité et la forme que doit prendre le processus de décision. Certains reproduisent un modèle hiérarchique, alors que d'autres privilégient une remise en question des rapports de pouvoir, y compris dans la structure interne de leur groupe, d'où la formule du collectif - ou collective - (Couillard et Côté, 1991). Certains groupes adoptent des formules mixtes résultant de la combinaison d'un conseil d'administration formel utilisé de manière accessoire (signer les demandes de subventions, notamment) avec un mode de fonctionnement en collectif et des prises de décisions au consensus dans le quotidien.

La réussite d'un partenariat exige un minimum de compatibilité entre les structures qui se rencontrent. Les difficultés les plus sérieuses se posent lorsque la logique bureaucratique, par définition hiérarchisée et orientée vers la réalisation d'objectifs précis, se voit remise en question par un fonctionnement basé sur le collectif et le consensus et visant un développement global de l'être humain. Les groupes qui privilégient un tel mode d'organisation ont l'impression de poser problème aux intervenantes du réseau officiel qui ont alors à composer avec l'ensemble des membres plutôt qu'avec une personne déléguée. Là où le collectif accepte le compromis et délègue une représentante, celle-ci bénéficie rarement d'une quelconque marge de manœuvre lorsque vient le temps de prendre position. Elle doit alors retourner au groupe pour consultation, ce qui, du point de vue de l'appareil d'État, alourdit grandement les négociations et le processus de décision et nuit à l'efficacité tant souhaitée. Ainsi, en exigeant que chaque groupe communautaire relève d'un 
conseil d'administration formel, l'État tente d'éliminer ce qu'il considère comme un problème d'efficacité et ce, sans se soucier de la nécessaire restructuration des rapports de pouvoir à l'interne.

La réforme Côté n'est donc pas sans conséquence sur la vie et le fonctionnement interne des groupes. À long terme, certains d'entre eux seront légitimés, alors que d'autres vont être marginalisés parce qu'ils sont trop lourds à porter en tant que partenaires. On voit clairement ici qu'en dépit d'une volonté d'ouverture et de respect, certaines contraintes que l'on pourrait qualifier de structurelles se mettent en place pour homogénéiser les partenaires ${ }^{13}$.

La motivation et la formation des ressources humaines sont très diverses et viennent à leur tour complexifier le paysage. Dans les groupes de femmes, on trouve des bénévoles mues par la bonne volonté et un désir réel d'apporter leur contribution à l'allégement des problèmes que vivent les femmes, des militantes politiques féministes déterminées à lutter contre le patriarcat sous toutes ses formes, aussi bien que des femmes intéressées avant tout à gagner leur vie en devenant permanentes rémunérées. Certaines femmes partent de leur expérience personnelle pour intervenir, d'autres se donnent des formations complémentaires, très souvent à caractère féministe. D'autres encore ont leur diplôme universitaire et un savoir livresque à monnayer.

Le développement d'un partenariat suppose la création de ponts avec des représentantes des groupes, et il semble certain que ceux-ci seront très différents selon la personne choisie pour assumer ce rôle. Là où un vocabulaire commun est un atout, certaines femmes sont très évidemment en position de force dans les rapports externes, sans nécessairement avoir des assises solides à l'interne, ce qui risque de compromettre le partenariat qu'elles doivent pourtant contribuer à consolider.

Le soutien financier contribue lui aussi aux différences entre les groupes. L'État, notamment par l'intermédiaire des ministères de la Santé et des Services sociaux et de l'Éducation, est le principal bailleur de fonds des groupes de femmes. Ce soutien financier ne vient pas sans contraintes et, c'est bien connu, les groupes ajustent leur programmation selon les priorités de l'État afin de garantir l'arrivée de fonds nécessaires à leur continuité. Dans ce processus d'ajustement, seule une partie des groupes bénéficie d'avantages sur les autres. Ces groupes deviennent alors des partenaires de choix. En retour, un financement moins précaire entraîne une plus grande stabilité du personnel qui peut alors se permettre d'approfondir certains dossiers et de là mieux asseoir son expertise. La reconnaissance de cette expertise en fera alors un partenaire encore plus sollicité.

13. À ce sujet, on prévoit même créer une "culture commune » entre les représentants des institutions du réseau de la santé et des services sociaux et ceux des groupes communautaires. 
Quelques groupes échappent en partie à ces contraintes en bénéficiant d'un soutien indépendant de l'État (des communautés religieuses par exemple), du moins pour le fonctionnement. Il serait intéressant de voir si ces groupes deviennent des partenaires, et à quelles conditions ils contribuent à l'interface.

Enfin, l'origine du groupe peut aussi constituer un élément de différenciation. Selon que le groupe résulte de l'intervention de religieuses déchargées par leur communauté pour travailler auprès des démunies, de femmes qui ont décidé de se prendre en charge ou de l'impulsion d'une organisatrice communautaire du CLSC qui voit là un moyen de réaliser ses objectifs, les liens avec le réseau et l'appareil d'État risquent d'être très différents. Là où les intervenantes communautaires du CLSC agissent comme conseillères, elles renforcent nécessairement les liens avec le réseau. En revanche, une identification trop étroite entre un groupe et un CLSC, ou une communauté religieuse, peut avoir, dans certains cas, un effet pervers. Il peut alors devenir difficile, pour le groupe, d'être reconnu en tant qu'acteur légitime du mouvement communautaire (qui possède ses propres critères de reconnaissance). Dans les cas extrêmes, il peut même être perçu comme l'instrument d'un pouvoir autre.

\section{Les liens inter-organisationnels}

L'hétérogénéité des groupes de femmes non seulement se manifeste dans des caractéristiques internes, mais elle s'inscrit aussi dans la diversité des liens inter-organisationnels entretenus par chacun. C'est d'abord dans leur propre bassin qu'ils puisent, mais aussi dans l'ensemble des organismes du milieu. On ajoutera aussi les contacts avec les représentants des divers paliers de gouvernement (conseiller, maire, député provincial, député fédéral et ministre) ainsi que les liens plus étroits avec les institutions du réseau de la santé et des affaires sociales. Les ponts créés avec les CLSC ne constituent donc qu'un seul aspect de ce réseau externe plus ou moins complexe selon les groupes.

Ces liens, peu importe le partenaire, suivent deux modèles: le premier implique une relation duale entre un groupe et un partenaire (un centre d'accueil pour femmes et un CLSC, par exemple). Le second découle de pratiques de collaboration liées à des lieux de concertation (notamment les tables de concertation, les regroupements et les réseaux).

Nous n'insisterons pas sur le premier modèle, puisqu'il a déjà fait l'objet de nombreuses analyses dont l'essentiel est repris dans des écrits sur le sujet. Rappelons simplement que ce modèle est très courant et que la presque totalité des groupes de femmes entretiennent des relations avec des partenaires divers. Cependant, le modèle qui suppose une concertation mérite une attention particulière, puisqu'il est souvent présenté comme le plus prometteur pour la création d'un partenariat entre le réseau et le communautaire. 
Précisons d'abord qu'il existe deux types de lieux de concertation où les groupes de femmes sont actifs. Le premier type fait référence aux regroupements ${ }^{14}$ composés exclusivement de groupes de femmes ${ }^{15}$ et qui sont associés à un espace territorial correspondant aux régions du Québec. Sur le territoire où se déroulent nos recherches, le Regroupement des groupes de femmes de la rive-nord (région 03, Québec métropolitain) est un exemple. Environ une quarantaine de groupes, sur un potentiel de 70 , sont présents au cours d'une année. Les thèmes discutés varient d'une rencontre à l'autre et, de l'avis des groupes participants, il s'agit là d'un lieu d'information, de ressourcement et d'échange d'expertise.

Le deuxième type de lieux de concertation renvoie aux tables de concertation axées sur des problématiques particulières, dans lesquelles les groupes de femmes s'associent à d'autres intervenants venant autant du milieu que du réseau et ce, à un niveau local ou sous-régional. Ici, les groupes de femmes sont des partenaires au même titre que les autres groupes communautaires ou que les ressources institutionnelles affiliées qui siègent tous autour d'une même table. À titre d'exemple, mentionnons la Table de concertation des organismes travaillant en violence conjugale et le Regroupement des ressources non institutionnelles en santé mentale, tous deux du Québec métropolitain (Couillard et Côté, 1991).

Bien qu'ils soient reconnus comme étant des lieux favorables à la consolidation de l'interface, les regroupements et les tables de concertation ne sont pas sans poser problèmes. Rappelons que l'un des objectifs implicites de ces lieux est de créer une culture commune propice à la communication et permettant le partage d'informations, la formation des participantes ou l'élaboration d'actions communes. Un tel projet ne peut être mis en œuvre sans une bonne dose de compromis, et nos données montrent que les valeurs et les idéaux politiques des membres en présence sont parfois si éloignés que toute tentative d'harmonisation mène à un cul-de-sac. De plus, la nécessité de partager des ressources financières limitées crée une situation paradoxale: alors que le lieu doit être un lieu de partage et de soutien, il s'avère que, parfois, la défense des intérêts de son groupe prime sur la solidarité.

14. On ne fait pas référence ici aux regroupements provinciaux, tels Le Regroupement des centres de femmes du Québec ou encore le Regroupement des maisons d'hébergement du Québec.

15. Le Québec est la seule province à fonctionner selon ce modèle. Dans les autres provinces, on trouve plutôt des coalitions provinciales ad hoc. Au Québec, par contre, on compte une douzaine de ces tables (appelées parfois aussi réseau ou regroupement), réparties selon les régions administratives de la province. Ces tables ont toutes été créées entre 1980 et 1990 (la moitié après 1985). Leurs activités sont financées par le Secrétariat d'État (fédéral) et, dans leur ensemble, elles représentent environ 320 groupes de femmes. Chacune de ces tables se réunit à un rythme d'environ quatre fois par an. 
Des groupes porteurs de valeurs et d'intérêts divergents se retrouvent donc à la même table, représentés par des personnes qui elles-mêmes se situent différemment selon qu'elles sont professionnelles, permanentes rémunérées ou militantes bénévoles. Ces différences sont la plupart du temps latentes ou réprimées à des fins de compromis. Elles émergent cependant avec force lorsqu'une demande est soumise au groupe soit pour un appui, soit pour qu'il en arrive à une position claire sur un sujet précis, surtout si celuici a des connotations politiques qui l'associent à une tendance perçue comme radicale. Dans certains cas, des conflits plus ou moins ouverts, voire des démissions et des retraits suivent de telles tentatives. L'existence même de la Table est alors menacée et seul un travail patient de diplomatie peut ramener les parties à travailler ensemble.

Préserver une certaine harmonie, ne pas «faire éclater » le groupe deviennent ainsi des préoccupations constantes, sinon de toutes les représentantes, du moins de celles qui croient au bien-fondé de tels lieux de concertation. II en résulte alors une pression plus ou moins tacite en faveur d'un nivellement des positions idéologiques et des discours afin d'éviter de choquer, de polariser et de démobiliser. C'est là le prix à payer pour la concertation. Cependant, les représentantes de factions diamétralement opposées (féministes radicales et déléguées de groupes conservateurs, par exemple) s'interrogent régulièrement sur les limites acceptables au-delà desquelles elles perdraient leur légitimité vis-à-vis de leur groupe et de leurs pairs. Dans un tel contexte, ce sont clairement les groupes aux positions centristes et modérées qui l'emportent, puisque pour eux le coût du compromis est visiblement moindre. En plus de donner leur couleur à la Table, ils se placent avantageusement dans l'éventualité d'un partenariat.

Si le compromis sur le plan des valeurs et des idéaux peut être menaçant pour certains, le fait de partager de l'information, surtout celle qui facilite l'accès à des fonds, n'est pas sans poser quelques pièges. C'est ainsi qu'une des raisons d'être de ces lieux devient un outil à double tranchant pour bien des groupes. Cette démocratisation contrecarre parfois des stratégies qui s'appuieraient autrement sur des relations personnelles, un lobby exigeant ou des filières privées. Par contre, la peur d'être tenus à l'écart d'informations pertinentes incite la plupart à participer aux réunions et à prendre le risque du contact avec les autres.

II ne fait aucun doute que ces lieux de concertation constituent des espaces privilégiés pour créer des alliances et négocier avec des partenaires mieux nantis ou jouissant d'une position de force. Ce sont donc des motifs «politiques » qui rassemblent les groupes et non pas simplement des préoccupations financières. Mais il ne faut pas sous-estimer les tensions qui les habitent et ne jamais tenir pour acquise la concertation qui s'en dégage et qui est sans cesse à recréer. 


\section{Le roulement}

Jusqu'ici nous avons souligné les éléments qui contribuent à faire du communautaire une entité hétérogène. Ces éléments servent ici de points de repère afin de comprendre comment certains groupes sont privilégiés pour la création d'une interface, alors que d'autres sont marginalisés par l'intervention de I'État. Paradoxalement, tous les groupes que nous avons étudiés ont un trait commun qui, lui aussi, peut poser un défi à la réussite du partenariat. II s'agit du roulement dans le personnel bénévole et rémunéré et dans la clientèle desservie. Ce roulement joue sur deux registres: I'un horizontal, l'autre vertical. À I'horizontal, le roulement des bénévoles entraîne une mobilité inter-groupes, non seulement entre les groupes de femmes, mais aussi avec les autres groupes du communautaire. Sur le plan vertical, la mobilité se joue du communautaire vers des postes dans le réseau ou d'autres instances offrant des positions à caractère plus permanent. La clientèle desservie connaît elle aussi un roulement important, ce qui l'amène à côtoyer divers groupes, d'allégeances parfois très différentes, tout autant qu'à consulter les services offerts par le réseau.

Cette fluidité ne serait pas problématique si les réseaux de soutien dans lesquels sont imbriqués les groupes n'étaient pas le résultat de démarches personnelles. À l'heure actuelle, le rôle des individus est central dans la création et le maintien de réseaux d'échange et de groupes de pression. Confrontés à la fluidité du membership, les groupes de femmes sont donc appelés à constamment re-tisser les liens avec leurs vis-à-vis dans l'interface et doivent sans cesse initier des acteurs qui, sans être complètement nouveaux, exercent des fonctions différentes.

Cette mobilité a aussi un effet sur la mémoire collective des groupes qui répètent souvent les mêmes démarches en ignorant le travail déjà effectué par d'autres qui ont quitté sans laisser de trace, la pratique communautaire n'étant pas nécessairement consignée dans l'écrit. On retrouve alors des situations où la seule personne permanente est celle qui occupe une position stable, soit l'organisatrice communautaire. C'est parfois elle qui doit raviver la mémoire des autres et assurer ainsi la pérennité du groupe. Un tel rôle n'est pas sans créer des ambiguïtés et certaines de ces femmes s'interrogent assidûment sur la place qu'elles occupent dans l'organisme qu'elles ont l'impression de "porter à bout de bras ». Du point de vue du groupe, la situation est aussi problématique, car un rapport de dépendance s'installe alors qui va à l'encontre des idéaux défendus.

\section{CONCLUSION}

En conclusion, les propositions de la réforme Côté en ce qui concerne le communautaire ne sont pas sans susciter des inquiétudes du point de vue des 
groupes de femmes. La revue de la littérature révèle que ce sont surtout les difficultés liées à l'existence d'une interface qui sont mises en évidence. Certains auteurs sont plus optimistes et proposent que des attitudes de respect et d'ouverture seraient garantes de son succès.

Nous avons voulu montrer qu'en dépit de leur bien-fondé ces attitudes doivent se déployer dans un contexte où des éléments structurants à l'interne et à l'externe colorent les conditions dans lesquelles le communautaire se réalise. Nous avons vu que l'ensemble des groupes qui constituent le communautaire «féminin » est très hétérogène. Des facteurs comme le type d'activités et de services, les approches privilégiées, le mode d'organisation, la motivation et la formation des ressources humaines, les sources de financement, voire l'origine même du groupe servent aux permanentes et aux bénévoles à se situer les unes par rapport aux autres. Car les enjeux varient grandement en fonction du profil du groupe. C'est ainsi que les éléments créateurs de solidarité peuvent parfois être neutralisés par des éléments générateurs de tension, plaçant certains groupes dans une position avantageuse dans l'interface.

Nous avons aussi souligné la complexité des liens inter-organisationnels dans lesquels s'engagent les groupes de femmes. Ces liens sont de natures diverses, mais nous avons retenu la participation à des tables de concertation et à des regroupements comme étant porteuse de paradoxes importants. Ces lieux de représentation des intérêts propres à chacun sont aussi des occasions d'engager des négociations serrées entre groupes à propos de l'analyse de la conjoncture et de la définition des enjeux importants. Nous avons aussi souligné quelques-uns des effets non prévus entraînés par la mise sur pied de ces instances de concertation, notamment la valorisation des groupes centristes et de leur point de vue, ce qui n'est pas sans avoir des répercussions sur l'interface qui nous intéresse.

Enfin, le roulement dans le personnel bénévole et la clientèle a été évoqué comme un autre trait marquant de ces groupes, laissant certains sans mémoire collective et, par conséquent, sans enracinement profond.

En définissant quelques-uns de ces éléments, nous avons souligné l'importance d'appréhender le communautaire dans toute sa complexité, en tenant compte des contradictions et des tensions qui l'habitent. En réaffirmant son caractère polymorphe, nous voulons rappeler que la concertation exigée par le partenariat est sans cesse à renégocier et qu'elle ne peut en aucun cas constituer le point de départ d'un processus d'arrimage aux institutions étatiques. 


\section{Références bibliographiques}

ANAdON, M., D. MASSON, M. TRemblay et P.-A. Tremblay (1992). De l'organisation aux pratiques d'autodéveloppement: portrait des groupes de femmes au Saguenay-Lac-Saint-Jean. Université du Québec à Chicoutimi.

ANADON, M., D. MASSON, M. TREMBLAY et P.-A. TREMBLAY (1990). "Les collectives de femmes: une démocratie sororale», Nouvelles pratiques sociales, vol. 3, $\mathrm{n}^{\circ} 2: 57-70$.

ASSEMBlÉE NATIONALE (1991). Projet de loi 120 - Loi sur la santé et les services sociaux et modifiant diverses dispositions législatives. Québec: Éditeur officiel du Québec.

BÉLANGER, J.-P. (1987). La concertation dans le réseau des services de santé et des services sociaux au Québec. Dossier thématique présenté à la Commission d'enquête sur les services de santé et les services sociaux. Québec: Les Publications du Québec.

BOZZINI, L. (1990). "Rapport Rochon et Orientations Lavoie-Roux: une lecture plus optimiste ». Nouvelles pratiques sociales, vol. 3, n 1 : 107-120.

CAillouette, J. (1992). "La réforme Côté ou l'ambivalence de l'État à l'égard du communautaire», Service social, vol. 41, n 2: 115-129.

COHEN, Y. (1990). Femmes de parole: I'histoire des Cercles de fermières du Québec 1915-1990. Montréal: Le Jour.

COMMISSION D'ENQUÊTE SUR LES SERVICES DE SANTÉ ET LES SERVICES SOCIAUX (1988). Rapport. Québec: Les Publications du Québec.

CÔTÉ, G. (1991). Centre-femmes d'aujourd'hui, un portrait descriptif. Sous la direction de Marie-Andrée Couillard. Document reproduit manuellement.

COUILLARD, M.-A. (à paraître). «From Women's Point of View: Practicing Anthropology in a World of Differences. " Contribution à un ouvrage collectif sous la direction de Sally Cole et Lynne Philipps.

COUILLARD, M.-A et G. CÔTÉ (1992). «L'engagement des Québécoises : trajectoires identitaires. »Communication présentée dans le cadre de la Conférence annuelle de l'ACSALF, Montréal, acceptée pour publication dans les actes du colloque.

COUILLARD, M.-A et G. CÔTÉ (1991). «Se reconnaître et/ou se faire reconnaître. Réflexions sur le pouvoir dans des groupes de femmes de Québec. »Communication présentée dans le cadre du colloque de l'Institut canadien de recherche sur les femmes, Edmonton, Alberta.

COUILLARD, M.-A. (1990). «Bénévolat ou militance, simple question de terminologie? Les défis méthodologiques d'une incursion à la base. »Conférence présentée dans le cadre de l'ACFAS, Sherbrooke.

DAVID, F. (1991). La réforme Lavoie-Roux-Côté - Entre la vertu et la pratique... tout un océan d'incertitudes. L'R des centres de femmes du Québec et le Regroupement des ressources alternatives en santé mentale. Document reproduit manuellement.

DESLAURIERS, J.-P. (1991). "Quelques enjeux de la réforme Côté », Nouvelles pratiques sociales, vol. 4, $\mathrm{n}^{\circ} 1: 1-7$.

DUMAIS, A. (1991). Les CLSC et les groupes communautaires: un aperçu de leur collaboration. Centre de recherche sur les services communautaires, SainteFoy: Université Laval. 
DUMONT, M., M. JEAN, M. LAVIGNE ET J. StOdDART (1992). L'histoire des femmes du Québec depuis quatre siècles. Montréal : Le Jour.

FOURNIER, J. (1991). "La réforme Côté: le passage du local au régional », Inter-action communautaire, vol. 5, $\mathrm{n}^{\circ} 2: 10-12$.

FOURNIER, J. et D. BOURQUE (1990). «Réforme de la santé et des services sociaux: alliances inattendues entre certains acteurs?», Nouvelles pratiques sociales, vol. $3, \mathrm{n}^{\circ} 2$ : 173-180.

GOdBOut, J., M. LeDuC et J.-P. COLLIN (1987). La face cachée du système, rapport de recherche $\mathrm{n}^{\circ} 22$ présenté à la Commission d'enquête sur les services de santé et les services sociaux. Québec: Les Publications du Québec.

GuAY, L. (1991). Le choc des cultures: bilan de l'expérience de participation des ressources alternatives à l'élaboration des plans régionaux de services en santé mentale. Document de travail.

HOME, A.M. (1987). Les femmes, le sexisme et les petits groupes: réflexion sur la théorie des groupes. Québec: les cahiers de recherche du GREMF (Groupe de recherche multidisciplinaire féministe). Université Laval.

LAMOUREUX, J., M. GÉLINAS et K. TARI (1993). Femmes en mouvement. Trajectoires de l'Association féminine d'éducation et d'action sociale, AFEAS 1966-1991. Montréal : Boréal.

LAMOUREUX, J. et F. LESEMANN (1987). Les filières de l'action sociale - les rapports entre les services sociaux publics et les pratiques communautaires. Rapport présenté à la Commission d'enquête sur les services de santé et les services sociaux. Québec: Les Publications du Québec.

LAROCHelle, G. et S. RobiChaud (1991). «Contestation et attestation étatique. Le nouveau catéchisme des groupes communautaires au Québec », Revue française de science politique, vol. 4, $\mathrm{n}^{\circ} 5$ : 649-675.

LATÉRIÈRE, H. et L. VOYER (1991). Analyse préliminaire du livre blanc du ministère de la Santé et des Services sociaux- "La réforme de la santé et des services sociaux». Gouvernement du Québec, Conseil du statut de la femme.

MASSON, D., M. TREMBLAY et P.-A. TREMBLAY (1989). "Femmes et pratiques d'autodéveloppement en région: perspectives de recherche », Recherches féministes, vol. 2, $\mathrm{n}^{\circ} 1: 69-84$.

MONET-ChARTRAND, S. (1990). Pionnières québécoises et regroupements de femmes d'hier à aujourd'hui. Montréal : Les éditions du remue-ménage.

OuELLETTE, F.-R. (1986). Les groupes de femmes du Québec en 1985 : champs d'intervention, structures et moyens d'action. Québec: Conseil du statut de la femme.

PANET-RAYMOND, J. et D. BOURQUe (1991). Partenariat ou pater-nariat? La collaboration entre établissements publics et organismes communautaires oeuvrant auprès des personnes âgées à domicile. Université de Montréal, groupe de recherche en développement communautaire.

PARAZELLI, M. (1990). «Pour ajouter de la misère à la vie - L'impact d'une épidémiologie sociale étatique sur l'action communautaire et les problèmes sociaux », Service social, vol. 39, $\mathrm{n}^{\circ} 2$ : 175-187.

ROBERT, L. (1989). "Le partenariat entre le réseau institutionnel et la communauté: un paradigme à définir », Nouvelles pratiques sociales, vol. 2, nº 1:37-52. 
TABLE DES ORGANISMES COMMUNAUTAIRES ET BÉNÉVOLES (1991). De la reconnaissance à l'intégration proposée... une question de survie pour l'ensemble du mouvement communautaire et/ou bénévole. Position du mouvement communautaire et bénévole sur la réforme Côté.

TROTTIER, S. (1991). «La réforme Côté et les organismes communautaires », Nouvelles pratiques sociales, vol. 4, $\mathrm{n}^{\circ} 1: 147-152$.

VAILlancourt, Y., D. BOURQUE, F. DAVID et E. Ouellet (1987). La privatisation des services sociaux, Québec: Les Publications du Québec. 